\title{
The Portuguese Meeting in Biomathematics
}

\author{
Delfim F. M. Torres ${ }^{1, *}$, Iván Area ${ }^{2}$, César Silva ${ }^{3}$, Cristiana J. Silva ${ }^{1}$ \\ ${ }^{1}$ Center for Research and Development in Mathematics and Applications (CIDMA), \\ Department of Mathematics, University of Aveiro, 3810-193 Aveiro, Portugal \\ ${ }^{2}$ Departamento de Matemática Aplicada II, Universidade de Vigo, Galicia, Spain \\ ${ }^{3}$ CMA and University of Beira Interior, Portugal
}

\begin{abstract}
The main contributions of [Stat. Optim. Inf. Comput. Vol. 7, No. 3 (2019)], consisting of 7 papers selected and revised from the 2nd Portuguese Meeting in Biomathematics (EPB'2018), are highlighted.

Keywords Population dynamics; Competition; Stochastic differential equations; Optimal control; Gompertz models; contagion; Epidemiological and biomedical models; Lyapunov functionals; tuberculosis; Compartmental mathematical models; Fractional calculus; Global stability; delays.
\end{abstract}

DOI: $10.19139 /$ soic.v7i3.829

\section{Introduction}

Biomathematics is a hot emerging field that has just had a special year: the year of 2018 was dedicated to the interface of mathematics and biology. Because 2018 was the year of mathematical biology, with Portugal at the center of attentions having the main event of the European Mathematical Society (EMS) and the European Society for Mathematical and Theoretical Biology (ESMTB) being organized in Lisbon, the subject saw many activities scheduled during all year and spanning a wide range of current aspects of interest in mathematical biology. Here we report some of the main contributions of the 2nd Portuguese Meeting in Biomathematics (EPB'2018), which was held at University of Aveiro, Department of Mathematics, July 19 and 20, 2018 [2].

The Encontro Português de Biomatemática (Portuguese Meeting in Biomathematics) was initiated in 2016 at the Department of Mathematics of University of Beira Interior [1], with the objective of joining every two years the Portuguese researchers interested in biomathematics and at the same time to promote the interaction between mathematicians working on models arising from the area of biology with other researchers working on areas of biology that use mathematics as an important tool in their research. During the past years, BioMathematics has become a recognized important area of applied mathematics, where Statistics, Optimization and Information Computing have a crucial role. We wish all readers to enjoy our Special Issue (SI). Each one of these papers has been carefully scrutinized, having passed the standard refereeing process. Our editorial task was made lighter by the service of unrewarded but dedicated referees: we offer them our heartfelt thanks. We take also this opportunity to thank again all the contributors and participants of EPB'2018, the authors for submitting their work for possible publication, and the members of the Organizing Committee, for a splendid organization, at all levels. Thanks to the work of all, the meeting was unique and unforgettable.

*Correspondence to: delfim@ua.pt (Delfim F. M. Torres).

ISSN 2310-5070 (online) ISSN 2311-004X (print)

Copyright (C) 2019 International Academic Press 


\section{Contents}

This SOIC special issue comprises seven original research articles that were carefully selected among works presented at EPB'2018:

1. Harvesting in a random varying environment: optimal, stepwise and sustainable policies for the Gompertz model, by N. M. Brites and C. A. Braumann;

2. Analysis of a SIRI epidemic model with distributed delay and relapse, by A. Elazzouzi, A. Lamrani Alaoui, M. Tilioua and D. F. M. Torres;

3. The influence of reduced light on competing zooplanktonic populations - Inference from mathematical modeling and field data, by V. Irenze, M. Battuello, R. Mussat Sartor, N. Nurra and E. Venturino;

4. The risk of contagion spreading and its optimal control in the economy, by O. Kostylenko, H. S. Rodrigues and D. F. M. Torres;

5. A minimal HIV-AIDS infection model with general incidence rate and application to Morocco data, by E. M. Lotfi, M. Mahrouf, M. Maziane, C. J. Silva, D. F. M. Torres and N. Yousfi;

6. Numerical simulation of excitation-contraction in isolated cardiomyocytes, by J. A. Rodrigues and J. Oliveira;

7. Optimal control and sensitivity analysis of a fractional order TB model, by S. Rosa and D. F. M. Torres.

Next, we briefly describe the main contributions of them.

\subsection{Fish populations with natural growth}

Brites and Braumann consider a fish population with natural growth following a Gompertz model with harvesting proportional to population size and to the exerted fishing effort. Using stochastic control theory, they present numerical comparisons between three harvesting policies: the optimal policy with variable effort, the sub-optimal policy with stepwise variable effort, and the optimal sustainable policy with constant effort. The comparisons were realized in terms of the expected accumulated discounted profit in a finite time interval. The main result of this work is the fact that the optimal sustainable policy produces a slight smaller profit in comparison with the optimal policy, providing, however, a much steadier profit.

\subsection{Epidemic model with distributed delay and relapse}

Elazzouzi et al. investigate the global behaviour of an epidemic model with distributed delay and relapse. From the theory of functional differential equations with delay, they prove that the solution of the system is unique, bounded, and positive, for all time. The basic reproduction number for the model is computed and, by means of the direct Lyapunov method and LaSalle invariance principle, they investigate the global asymptotic stability of the equilibria.

\subsection{Light-influenced zooplankton model}

In the paper of Irenze et al., a mathematical model that describes the zooplankton spatio-temporal dynamics is analysed. The phytoplanktonic resource, not explicitly expressed in the model, is assumed to be unlimited, while the influence of phytoplankton is expressed by the light absorption, obtained experimentally, along the entire water column. The model exhibits four feasible equilibrium points and, for these points, the stability conditions and the possible local bifurcations are determined.

\subsection{Financial contamination as an epidemiological process}

In the work of Kostylenko et al., the process of financial contamination is investigated from the point of view of infection spreading among countries, which form an interconnected closed-form system. Both continuous-time and a discrete-time (network) epidemiological model are considered. Application of optimal control theory shows the need of a thorough control of the quantity guaranteed loans and the financial stability of countries in order to avoid the negative effects of financial risks. 


\subsection{HIV/AIDS transmission}

Lotfi et al. propose a non-linear mathematical model, of a varying population size in a homogeneously mixed population, for HIV/AIDS transmission. The global dynamics of the model, with a general incidence function, is investigated. The general incidence function may depend on the susceptible and HIV-infected individuals with no AIDS symptoms, which are not under treatment and therefore transmit the HIV virus. For a carefully chosen incidence function, they show that the model describes very well the reality given by the data of HIV/AIDS infection in Morocco from 1986 to 2015.

\subsection{On cardiac cells}

Rodrigues and Oliveira investigate a finite element method for a mathematical model of excitation-contraction in isolated cardiomyocytes, relevant in cardiac diseases. The results from the studied cases highlight the T-tubules distribution importance along the cardiomyocyte and that the fact that cell deformation is higher near the T-tubule boundary. The results obtained with the proposed model are faithful to what is known to be true with cell's physiology and pathological conditions (hypocalcemia), providing a simplification of the mathematical context of the excitation-contraction in isolated cardiomyocytes.

\subsection{Non-integer order modelling and optimal TB control}

Finally, the paper of Rosa and Torres give a sensitivity analysis for the basic reproduction number of a known tuberculosis (TB) model, proving that the most important parameter to have into account is the natural death rate. Then, they apply optimal control to a fractional extension of the TB model, showing that treatment is effective on the reduction of infected individuals. A change in the value of the fractional order derivative order corresponds to substantial variations on the solutions of the fractional optimal control problem. They recommend the fractional (non-integer) order model when treatment is expensive, and the classical/standard model otherwise.

\section{Acknowledgments}

The authors are grateful to the support of the the University of Aveiro (UA), the Department of Mathematics of UA (DMat), and the Center for Research and Development in Mathematics and Applications (CIDMA), project UID/MAT/04106/2019 of the Portuguese Foundation for Science and Technology (FCT), and COST Action CA16227 - Investigation and Mathematical Analysis of Avant-garde Disease Control via Mosquito Nano-TechRepellents (WG2 on "Structured Models \& Optimal Control"). They are also grateful to the Organizing Committee, the Scientific Committee, and all the anonymous referees of the papers submitted for possible publication. Moreover, they deeply thank David Yu and SOIC for all the support to the Special Issue.

\section{REFERENCES}

1. The Portuguese Meeting in Biomathematics, University of Beira Interior, October 13 and 14, 2016. http://epb2016.ubi . pt

2. 2nd Portuguese Meeting in Biomathematics, University of Aveiro, July 19 and 20, 2018. http: / / epb2018. weebly. com 